\title{
Risk of Gastrointestinal Events in Patients with Rheumatoid Arthritis After Withdrawal of Rofecoxib
}

\author{
CARLO A. MARRA, LARRY D. LYND, LINDSEY COLLEY, STEPHANIE S. HARVARD, DIANE LACAILLE, \\ ERIN SCHWENGER, ASLAM H. ANIS, and JOHN M. ESDAILE
}

ABSTRACT. Objective. To examine the incidence of gastrointestinal (GI) events in patients with rheumatoid
arthritis (RA) after the removal of rofecoxib from the market.
Methods. Residents of British Columbia with a diagnosis of RA who were chronic users of
cyclooxygenase 2 (COX-2) inhibitors or nonselective nonsteroidal antiinflammatory drugs
(nsNSAID) as of September 30,2004 , were included. We studied the risk of GI events using inci-
dence rates and adjusted HR from Cox proportional hazards regression using time-dependent
covariates.

Results. The cohort comprised 4266 patients with a mean age of 60 years and over $72 \%$ women, of which $2034(48 \%)$ were classified as COX-2 inhibitor users and $2232(52 \%)$ as chronic nsNSAID users as of September 30, 2004. The 2 groups were well balanced on baseline covariates except for comorbid conditions. In the year following rofecoxib withdrawal, 174 patients $(5.5 \%)$ experienced 1 or more GI events, defined as a GI-related physician visit or hospitalization. There was no statistically significant increase in the risk of a GI event between those classified as a COX-2 inhibitor or nsNSAID user at the time of withdrawal (HR 1.03, 95\% CI 0.69-1.54). Considering the drug exposure at the time of the event, there was no increased risk of GI events associated with the use of either COX-2 inhibitors or nsNSAID, or with the use of oral corticosteroids, low-dose aspirin, or clopidogrel, after adjustment for potential confounders.

Conclusion. In this cohort, withdrawal of rofecoxib did not result in a significant increase in GI events among patients with RA. (First Release March 15 2012; J Rheumatol 2012;39:910-15; doi:10.3899/jrheum.110604)

Key Indexing Terms:

RHEUMATOID ARTHRITIS GASTROINTESTINAL RISK ROFECOXIB

From the Faculty of Pharmaceutical Sciences, University of British Columbia (UBC), Vancouver; Centre for Health Evaluation and Outcome Sciences, Providence Health Care Research Institute, Vancouver; School of Population and Public Health, UBC, Vancouver, British Columbia; Memorial University of Newfoundland, St. John's, Newfoundland and Labrador; and Department of Medicine and Community Health Sciences, University of Calgary, Calgary, Alberta, Canada.

C.A. Marra, PharmD, PhD, Associate Professor, Director, Collaboration for Outcomes Research and Evaluation (CORE), Faculty of Pharmaceutical Sciences, UBC; Research Scientist, Centre for Health Evaluation and Outcome Sciences, Providence Health Care Research Institute; L.D. Lynd, PhD, Associate Professor, Associate Director, CORE, Faculty of Pharmaceutical Sciences, UBC; Research Scientist, Centre for Health Evaluation and Outcome Sciences, Providence Health Care Research Institute; L. Colley, MSc, Statistician, CORE, Faculty of Pharmaceutical Sciences, UBC; S.S. Harvard, MSc, School of Population and Public Health, UBC; D. Lacaille, MD, MHSc, Associate Professor of Rheumatology, Faculty of Medicine, UBC; Research Scientist, Arthritis Research Centre of Canada, Vancouver; E. Schwenger, BSc (Pharm), PharmD, Memorial University of Newfoundland; A.H. Anis, PhD, Professor of Health Economics, Director, M.H.A. Program, SPPH, Director, Centre for Health Evaluation and Outcome Sciences, Providence Health Care Research Institute; J.M. Esdaile, MD, MPH, Arthur J.E. Child Chair in Rheumatology Research, Professor of Medicine and Community Health Sciences, University of Calgary, Professor of Medicine, UBC, Scientific Director, Arthritis Research Centre of Canada. Prof. Marra and Prof. Lynd contributed equally to this report. Address correspondence to Dr. L.D. Lynd, 2146 East Mall, Faculty of Pharmaceutical Sciences, University of British Columbia, Vancouver, British Columbia V6T 1Z3, Canada.E-mail: larry.lynd@ubc.ca Accepted for publication January 13, 2012.
Since the withdrawal from the market of rofecoxib on September 30, 2004, due to an increased risk of cardiovascular (CV) adverse events ${ }^{1}$, several studies have sought to describe population-level treatment changes among users of rofecoxib and other selective cyclooxygenase 2 (COX-2) inhibitors $2,3,4,5,6,7$. These studies suggest that the rofecoxib withdrawal resulted in a decrease in dispensing of all selective COX-2 inhibitors ${ }^{2,3,4,7}$ because more COX-2 users discontinued nonsteroidal antiinflammatory drug (NSAID) therapy completely or switched to a nonselective NSAID (nsNSAID) than switched to another selective COX-2 inhibitor ${ }^{5,6}$. Among all users of selective COX-2 inhibitors 1 year after the withdrawal of rofecoxib, Sukel and colleagues ${ }^{6}$ observed that although the greatest proportion $(43 \%)$ discontinued NSAID use altogether, a large proportion $(28 \%)$ switched to an nsNSAID. Among the users who switched to an nsNSAID, only half were also prescribed a proton pump inhibitor (PPI), indicating that the treatment change may have increased their vulnerability to a gastrointestinal (GI) adverse event. This pattern raises the concern that large-scale switching from selective COX-2 inhibitors to more gastrotoxic nsNSAID may lead to an increase in GI events at the population level.

To our knowledge, 4 studies have been published examPersonal non-commercial use only. The Journal of Rheumatology Copyright $@$ $\odot 2012$. All rights reserved. 
ining the effects of the withdrawal of rofecoxib on the rate of GI events at a population level ${ }^{8,9,10,11}$, yet only 1 of these studies evaluated the difference in risk for patients switching to an nsNSAID compared to another COX-2 inhibitor ${ }^{11}$. Schneeweiss and colleagues ${ }^{11}$ found a significant short-term increase in upper GI complications among rofecoxib users with osteoarthritis (OA) or rheumatoid arthritis (RA) who switched to an nsNSAID compared to those who switched to another COX-2 inhibitor. That study also showed a significantly higher proportion of rofecoxib users switched to an nsNSAID (17\%) 1 month after the withdrawal than did users of any selective COX-2 inhibitor $(9 \%)^{11}$.

Comparing the reports of Schneeweiss, et al ${ }^{11}$ and Sukel, et $a l^{6}$, it appears that the proportion of COX-2 inhibitor users who switched to an nsNSAID may be lower within the OA/RA population than in the general population $(9 \%$ versus $26 \% 6$ at 1 month). Potential differences in NSAID switching patterns between all users of selective COX-2 inhibitors and users with OA or RA suggest that the influence of NSAID switching on GI outcomes may vary depending on the population. The purpose of our study was to describe postwithdrawal changes in the use of selective COX-2 inhibitors and nonselective NSAID among patients with RA specifically. Importantly, RA patients are at an increased risk of both $\mathrm{CV}$ and GI adverse events, making any NSAID use challenging. Further, exposure to specific NSAID may change frequently during followup among patients with RA, making time-dependent measures of NSAID exposure, and exposure to other drugs influencing GI risk, especially important. While Schneeweiss and colleagues ${ }^{11}$ tracked only the first treatment change within the 4 weeks following the withdrawal of rofecoxib and limited the followup of GI outcomes to 3 months, there is evidence of significant differences in treatment patterns 1 month and 1 year following the rofecoxib withdrawal ${ }^{6}$. Our study was therefore designed to characterize the risk of GI events in the RA population in the 12 months following the withdrawal of rofecoxib.

\section{MATERIALS AND METHODS}

Study design. A population-based retrospective cohort study using linked health database records of patients with RA in British Columbia (BC), Canada.

Data sources. Data on patient characteristics and health services use were obtained from the BC Medical Services Plan and Hospital Separations databases, which contain billing information on all outpatient physician visits and hospital discharges for the province of $\mathrm{BC}$. Data on drug use were obtained from the BC PharmaNet database, which contains a record of the specific drug, dispensation date, and days of medication supplied for every prescription dispensed in BC. Data from these 3 databases were linked for each individual patient using a unique identifying number derived from their Personal Health Number.

Inclusion/exclusion criteria. The study cohort was derived from a population-based cohort of patients with RA from all included RA cases $\geq 18$ years of age in the province of $\mathrm{BC}$ who had received care for their RA between 1996 and 2006, identified using physician billing data as described. We selected RA cases who had at least 1 physician visit for RA
[International Classification of Diseases, 9th revision (ICD-9) code 714.X] between September 30, 2002, and September 30, 2004, and who were chronic users of a COX-2 inhibitor or nsNSAID. Chronic users were defined as patients who had filled a prescription for a selective COX-2 inhibitor or nsNSAID and who met the following criteria: (1) were taking a COX-2 inhibitor or nsNSAID as of September 30, 2004; and (2) received a supply of at least 180 days of either a COX-2 inhibitor or nsNSAID (or any combination thereof) between October 1, 2003, and September 30, 2004 (i.e., in the year prior to the withdrawal).

Sample characteristics. Patient characteristics, determined as of September 30,2004 , included age, sex, history of a previous GI event or visit to a GI specialist (practitioner's registered specialty code 56), and the number of chronic diseases identified between October 1, 2003, and September 30, 2004. The Deyo-adapted Charlson Index was used to determine the number of comorbid diseases for each patient ${ }^{12,13,14}$. Patients with 2 or more comorbid diseases were categorized as 1 group because of the small number of patients with comorbidities in this range ${ }^{12,15}$. Every patient was classified as a user of either a selective COX-2 inhibitor or nsNSAID based on the most recent dispensed prescription prior to September 30, 2004.

Followup. Patients were followed from the date of their last day's supply of rofecoxib (rofecoxib users) or from October 1, 2004 (all other patients) until a GI event (hospitalization or physician visit), death, or September 30 , 2005, whichever occurred first. If a patient experienced both a physician visit and a hospitalization, the hospitalization was treated as the first event regardless of the order of events.

Assessment of drug exposure. For all drugs, exposures were assumed to begin on the dispensation date and the defined daily dose was used in the calculation of the number of days exposed to a drug. The exposure period for selective COX-2 inhibitors, nsNSAID, low-dose aspirin, PPI/misoprostol, $\mathrm{H}_{2}$-receptor blockers, and clopidogrel was defined as $125 \%$ of the number of days of medication supplied. Exposure to warfarin was assumed to be constant until a break of 3 months from the end of 1 prescription (defined as $125 \%$ of the number of days of medication supplied) and the dispensing of a subsequent prescription. As corticosteroids are supplied in a variety of strengths (e.g., for prednisone, $1 \mathrm{mg}, 5 \mathrm{mg}$, and $50 \mathrm{mg}$ tablets) and dosage changes may occur that are not recorded in PharmaNet, the exposure period for corticosteroids was defined as 45 days from the dispensation date if no further corticosteroids were dispensed. If a prescription for a selective COX-2 inhibitor was dispensed while a patient had a supply of an nsNSAID it was assumed that the nsNSAID was stopped and the patient switched to a COX-2 inhibitor, and vice-versa. If both the number of days prescribed was fewer than 7 and the quantity dispensed of a COX-2 inhibitor or nsNSAID was to last $<7$ days, it was assumed that a mistake had been made in the data entry and the prescription was excluded from the analysis (resulting in exclusion of $0.4 \%$ of COX-2 and nsNSAID prescriptions).

Outcome measures. The primary outcome was the occurrence of a GI event potentially related to NSAID use such as ulcerations, perforations, or bleeds in the upper or lower GI tract requiring hospitalization or physician visit. If a patient experienced both a GI-related physician visit and hospitalization, the hospitalization was treated as the GI event regardless of the order of events. GI hospitalizations were identified from the Hospital Separations database and included diagnoses of ulceration, perforation, or bleeding in the upper GI tract (ICD-9 codes 531.x, 532.x, 533.x, 534.x, $535.01,535.31,578.0,578.1,578.9,537.83)$ or of a lower GI event occurring in either the small intestine (beyond the duodenum) or the colon/rectum, i.e., gross rectal bleeding, lower GI perforation, ulceration, or diverticulitis with hemorrhage (ICD-9 codes 562.02, 562.03, 562.12, 562.13, $569.3,569.41,569.83,569.82,569.85)$. Hospitalizations for operative gastroscopy or other invasive GI diagnostic procedures were also included (ICD-9 codes 55.0, 56.83, 56.89). Physician visits for GI events were identified from the BC Medical Services Plan physician billing data using the ICD-9 codes listed above or an appropriate fee-for-service code.

We also calculated the number of switches a patient made between 
valdecoxib, celecoxib, meloxicam, and other nsNSAID in the month and year following withdrawal (or the month following the end of a rofecoxib prescription or to the end of the followup period). For patients who made at least 1 switch from 1 NSAID to another, we identified the drug dispensed that represented the first switch in the study period.

Statistical analyses. Baseline characteristics of the sample were summarized using means and standard deviations (SD) for continuous data and frequencies and percentages for categorical data. The baseline differences between those classified as a selective COX-2 inhibitor and an nsNSAID user were investigated using 2-sample t tests and Pearson chi-square for continuous and categorical data, respectively. A Cox regression model with time-dependent covariates was used to compare HR for GI events between drug exposure categories. All drug exposures were considered as time-dependent variables in the model. At any given time, the model considered a patient as belonging to the risk sets corresponding to their drug exposure categories. GI event rates were adjusted for baseline patient characteristics that could be potential confounders, including age, sex, Charlson comorbidity index, classification of a selective COX-2 inhibitor or nsNSAID user as of September 30, 2004, and history of a GI event/visit between October 1, 2003, and September 30, 2004. Statistical significance was set at 0.05 (2-tailed). To assess the robustness of the results, the definitions of drug exposures were varied in 2 sensitivity analyses. These analyses considered all GI events that occurred within $100 \%$ and $150 \%$ of the number of days of medication supplied (as opposed to within $125 \%$ of days supplied in the main analysis). We also conducted a sensitivity analysis in which we limited the outcome to only the patients with hospitalizations after September 30, 2004.

\section{RESULTS}

Baseline patient characteristics. There were 19,085 individuals who fit our definition of RA who were prescribed either a selective COX-2 inhibitor or nsNSAID in the year prior and the year following September 30, 2004. Once the inclusion criteria for chronic use were applied, the cohort comprised 4266 patients, of which $2034(48 \%)$ were classified as COX-2 inhibitor users and $2232(52 \%)$ as nsNSAID users as of September 30, 2004. Baseline patient characteristics for COX-2 inhibitor and nsNSAID users are shown in Table 1. Among patients classed as COX-2 inhibitor users at the time of the withdrawal, the majority were taking celecoxib (52\%), followed by rofecoxib (37\%). There were no significant differences between COX-2 inhibitor and nsNSAID users for previous GI event $(\mathrm{p}=0.354)$; however, these groups did differ in the mean age $(p=0.032)$, sex $(p=$

Table 1. Baseline characteristics of COX-2 inhibitor and nsNSAID users. Data are mean \pm SD or no. (\%) unless otherwise indicated.

\begin{tabular}{|c|c|c|c|}
\hline Characteristics & $\begin{array}{c}\text { COX-2 Inhibitor, } \\
\mathrm{n}=2034\end{array}$ & $\begin{array}{l}\text { NSAID, } \\
\mathrm{n}=2232\end{array}$ & $\mathrm{p}$ \\
\hline Age, yrs & $60.3 \pm 13.4$ & $59.4 \pm 14.0$ & 0.032 \\
\hline Female* $(\%)$ & $1492(73.4)$ & $1575(70.6 \%)$ & 0.043 \\
\hline Previous GI event/visit (in prior year; \%) & $116(5.7)$ & $113(5.1)$ & 0.354 \\
\hline Charlson comorbidity index* $(\%)$ & & & $<0.001$ \\
\hline 0 & $1327(65.2)$ & $1289(57.8)$ & \\
\hline 1 & $498(24.5)$ & $691(31.0)$ & \\
\hline $2+$ & $209(10.3)$ & $252(11.3)$ & \\
\hline \multicolumn{4}{|l|}{ Drugs taken at withdrawal (\%) } \\
\hline Rofecoxib & $484(23.8)$ & & \\
\hline Rofecoxib + GI** & $155(7.6)$ & & \\
\hline Rofecoxib + anticoagulant/corticosteroid ${ }^{* * *}$ & $61(3.0)$ & & \\
\hline Rofecoxib + GI + anticoagulant/corticosteroid ${ }^{\dagger \dagger}$ & $47(2.3)$ & & \\
\hline Valdecoxib & $162(8.0)$ & & \\
\hline Valdecoxib + GI** & $27(1.3)$ & & \\
\hline Valdecoxib + anticoagulant/corticosteroid*** & $22(1.1)$ & & \\
\hline Valdecoxib + GI + anticoagulant/corticosteroid ${ }^{\dagger \dagger}$ & $12(0.6)$ & & \\
\hline Celecoxib & $645(31.7)$ & & \\
\hline Celecoxib + GI** & $231(10.4)$ & & \\
\hline Celecoxib + anticoagulant/corticosteroid $* * *$ & $117(5.8)$ & & \\
\hline Celecoxib + GI + anticoagulant/corticosteroid ${ }^{\dagger \dagger}$ & $68(3.3)$ & & \\
\hline Meloxicam & & $259(11.6)$ & \\
\hline Meloxicam + GI** & & $91(4.1)$ & \\
\hline Meloxicam + anticoagulant/corticosteroid $* * *$ & & $44(2.0)$ & \\
\hline Meloxicam + GI + anticoagulant/corticosteroid ${ }^{\dagger \dagger}$ & & $21(0.9)$ & \\
\hline Other nsNSAID & & $1112(49.8)$ & \\
\hline Other nsNSAID + GI** & & $425(19.0)$ & \\
\hline Other nsNSAID + anticoagulant/corticosteroid*** & & $155(6.9)$ & \\
\hline Other nsNSAID + GI + anticoagulant/corticosteroid ${ }^{\dagger \dagger}$ & & $122(5.5)$ & \\
\hline
\end{tabular}

* Significant differences between groups $(\mathrm{p}<0.05)$. ** Includes proton pump inhibitors $(\mathrm{PPI}) /$ misoprostol and $\mathrm{H}_{2}$ blockers. ${ }^{* * *}$ Includes clopidogrel, low-dose aspirin, oral corticosteroids, and warfarin. ${ }^{\dagger \dagger}$ Includes either PPI/misoprostol or $\mathrm{H}_{2}$ blockers and clopidogrel or low-dose aspirin or oral corticosteroids or warfarin. GI: gastrointestinal; nsNSAID: nonselective nonsteroidal antiinflammatory drug; COX-2: cyclooxygenase 2. 
0.043 ), and number of comorbid diseases based on the Charlson comorbidity index $(\mathrm{p}<0.001)$.

NSAID switching in the month following rofecoxib withdrawal. Table 2 shows the number of switches between valdecoxib, celecoxib, meloxicam, and other nsNSAID in the first month following rofecoxib withdrawal, or in the month following the end of a rofecoxib prescription after the date of withdrawal. Table 3 describes the first switch among patients who switched at least once in the month following withdrawal or the end of rofecoxib prescription. Among the 750 patients who had an active prescription for rofecoxib at the time of withdrawal, 263 did not use any NSAID in the month following the end of their prescription. Among the 487 who made a switch, over half switched to another COX-2 inhibitor (38\% switched to celecoxib and $27 \%$ to valdecoxib) rather than an nsNSAID (17\% switched to meloxicam and $18 \%$ to another nsNSAID). Among patients taking a COX-2 inhibitor other than rofecoxib at the time of the withdrawal, only a very small percentage switched to an nsNSAID in the following month. Specifically, $1 \%$ of patients switched from valdecoxib and celecoxib, respectively, to either meloxicam or another nsNSAID.

NSAID switching in the year following rofecoxib withdrawal. Table 4 shows the number of switches between valdecoxib, celecoxib, meloxicam, and other nsNSAID in the year following the withdrawal. Table 5 outlines the first switch among patients who switched at least once in the year following withdrawal. Among the 586 patients who were taking rofecoxib at the time of withdrawal and made a switch before September 30, 2005, over half switched to another COX-2 inhibitor (37\% to celecoxib and $24 \%$ to valdecoxib) as their first alternative antiinflammatory.

Gastrointestinal events. In the year following the withdrawal, 174 patients $(5.5 \%)$ experienced at least 1 GI event requiring a physician visit or hospitalization. This included 119 patients $(2.8 \%)$ who had a GI-related physician visit, 16 $(0.4 \%)$ who were hospitalized due to a GI event, and 39 $(0.9 \%)$ who had both a physician visit and a hospitalization. The results of the time-dependent multivariate Cox regression model are displayed in Table 6. In this analysis, the hazard of GI events between October 1, 2004, and September 30, 2005, was about 3 times higher among patients who experienced a GI event in the year prior to September 30, 2004, compared to those who did not (HR 3.35, 95\% CI 2.22-5.07). GI events were significantly more common among patients with 1 comorbid disease relative to those with no comorbid disease (HR 1.44, 95\% CI 1.03-2.01). There was no statistically significant increase in the risk of a GI event between those classified as using a COX-2 inhibitor versus nsNSAID users at the time of withdrawal (HR 1.03, 95\% CI 0.69-1.54). Further, considering the drug exposure at the time of the event, there was no increased risk of GI events associated with the use of either COX-2 inhibitors or nsNSAID, or with the use of oral corticosteroids, low-dose aspirin, or clopidogrel, after adjustment for potential confounders.

Table 2. Number of drug changes in month following withdrawal of rofecoxib ${ }^{\dagger}$.

\begin{tabular}{lcccc}
\hline & \multicolumn{2}{c}{ No. Drug Changes in Month Following Withdrawal } & Total \\
Drug at Withdrawal & 0 & 1 & 2 & $487^{\dagger \dagger}$ \\
\hline Rofecoxib & 0 & 461 & 26 & 223 \\
Valdecoxib & 212 & 11 & 0 & 1058 \\
Celecoxib & 1041 & 6 & 0 & 418 \\
Meloxicam & 412 & 19 & 2 & $1807^{*}$ \\
Other nsNSAID & 1786 & \\
&
\end{tabular}

Table 3. First change in drug in the month following withdrawal of rofecoxib.

\begin{tabular}{lccccc}
\hline \multicolumn{4}{c}{ Drug of 1st Switch in Month Following Withdrawal } & Other \\
Drug at Withdrawal & Valdecoxib & Celecoxib & Meloxicam & nsNSAID & Total \\
\hline Rofecoxib & 131 & 187 & 83 & 86 & 487 \\
Valdecoxib & 0 & 1 & 3 & 7 & 11 \\
Celecoxib & 5 & 0 & 1 & 11 & 17 \\
Meloxicam & 1 & 3 & 0 & 2 & 6 \\
Other nsNSAID & 10 & 8 & 3 & 0 & 21 \\
\hline
\end{tabular}

nsNSAID: nonselective nonsteroidal antiinflammatory drug. 
Table 4. Number of drug changes in year following withdrawal of rofecoxib ${ }^{\dagger}$.

\begin{tabular}{|c|c|c|c|c|}
\hline \multirow{2}{*}{$\begin{array}{l}\text { Antiinflammatory } \\
\text { at Withdrawal }\end{array}$} & \multicolumn{4}{|c|}{ No. Drug Changes in Year Following Withdrawal } \\
\hline & 0 & 1 & $2+$ & Total \\
\hline Rofecoxib & 0 & 344 & 224 & $568 *$ \\
\hline Valdecoxib & 86 & 116 & 21 & 223 \\
\hline Celecoxib & 844 & 154 & 61 & 1059 \\
\hline Meloxicam & 349 & 46 & 23 & 418 \\
\hline Other nsNSAID & 1740 & 37 & 36 & 1813 \\
\hline
\end{tabular}

$\dagger$ Or to September 30, 2005, for patients finishing rofecoxib prescription after October 1,2004 . * 2 patients had a gastrointestinal event before starting a new COX-2 inhibitor or nsNSAID prescription. nsNSAID: nonselective nonsteroidal antiinflammatory drug; COX-2: cyclooxygenase inhibitor.

Sensitivity analyses. Varying the time window of exposure from $100 \%$ to $150 \%$ of the days' supply did not affect the estimates of the HR for the multivariable COX regression, suggesting that the model and the results are robust to our assumption of sporadic use of antiinflammatory drug therapy (data not shown). Further, by limiting the outcome to only patients with a GI-related hospitalization after September 30, 2004, the results were consistent, with the exception of a decreased risk of GI events associated with COX-2 inhibitor use (HR 0.32, 95\% CI 0.12-0.84).

\section{DISCUSSION}

To our knowledge, this is the first study to assess the effect of the withdrawal of rofecoxib on GI events specifically in patients with RA, based on time-dependent drug exposures. The RA patient population is of particular interest given that NSAID are often used as a first-line treatment option in combination with disease-modifying antirheumatic drugs for RA, and patients with RA are already at an increased risk for both CV and GI events. In this retroprospective cohort, we found no statistically significant difference in GI events between patients who switched to an nsNSAID compared to those who switched to another COX-2 inhibitor in either the month or the year following the withdrawal of rofecoxib. In the year following the withdrawal, more than half the patients previously receiving rofecoxib switched to another COX-2 inhibitor, and the overall incidence of GI events was $5.5 \%$. This rate was significantly higher among patients who
Table 6. Cox regression model with time-dependent exposure to determine the association between drug exposure and gastrointestinal events.

\begin{tabular}{lccc}
\hline Variable & HR & $(95 \% \mathrm{CI})$ & $\mathrm{p}$ \\
\hline Nonselective NSAID & 0.72 & $(0.52-1.14)$ & 0.9530 .191 \\
COX-2 inhibitor & 0.67 & $(0.42-1.04)$ & 0.076 \\
Oral corticosteroid & 1.14 & $(0.74-1.75)$ & 0.553 \\
Warfarin & $1.72(0.80-3.70)$ & 0.168 \\
Low-dose aspirin & 1.55 & $(0.67-3.55)$ & 0.304 \\
Clopidogrel & $1.97(0.79-4.90)$ & 0.145 \\
Patient taking COX-2 inhibitor at & & & 0.869 \\
$\quad$ withdrawal & $1.03(0.69-1.54)$ & 0.602 \\
Female & $0.92(0.66-1.27)$ & $<0.001$ \\
GI event in prior 12 months & 3.35 & $(2.22-5.07)$ & 0.061 \\
Age (per additional 10 years) & $1.12(1.00-1.25)$ & \\
Charlson comorbidity index & & & 0.034 \\
$\quad$ (reference) & 1 & - & 0.110 \\
$\quad 1$ & 1.44 & $(1.03-2.01)$ \\
$\quad$ 2+
\end{tabular}

experienced a GI event in the year prior to September 30, 2005, than among those who did not. Taking into account drug exposure at the time of the event, no increased risk was found with concomitant corticosteroid use, low-dose aspirin, or clopidogrel, after adjustment for potential confounders.

The effect of the withdrawal of rofecoxib on GI outcomes in chronic COX-2 inhibitor users has been described in the general population and within a group of patients with OA or RA. Taha and colleagues noted a slight increase between 2002 and 2005 in the rate of GI bleeds in Scotland, yet they found this increase to be associated with an increase in the use of low-dose aspirin, other antithrombotics, and alcohol, and not significantly associated with nsNSAID use $^{8}$. In the United Kingdom, Wheeler and colleagues noted a slight increase in the rate of hospitalizations for GI hemorrhage after 2002, but noted that this predated the withdrawal of rofecoxib ${ }^{10}$. In an international comparison of mortality rates due to myocardial infarction and GI hemorrhage, Metcalfe, et al found no effect of the rofecoxib withdrawal on mortality, and in fact noted an apparent reduction in mortality due to GI hemorrhage and acute myocardial infarction after $2004^{9}$. Among patients with OA or RA, Schneeweiss, et al found no increase in the rates of GI-relat-

Table 5. First change in drug in the year following withdrawal of rofecoxib ${ }^{\dagger}$.

\begin{tabular}{|c|c|c|c|c|c|}
\hline \multirow[b]{2}{*}{$\begin{array}{l}\text { Antiinflammatory } \\
\text { at Withdrawal }\end{array}$} & \multicolumn{4}{|c|}{ Drug of 1st Switch in Year Following Withdrawal } & \multirow[b]{2}{*}{ Total } \\
\hline & Valdecoxib & Celecoxib & Meloxicam & $\begin{array}{c}\text { Other } \\
\text { nsNSAID }\end{array}$ & \\
\hline Rofecoxib & 136 & 208 & 98 & 126 & 568 \\
\hline Valdecoxib & 0 & 35 & 29 & 73 & 137 \\
\hline Celecoxib & 16 & 0 & 30 & 169 & 215 \\
\hline Meloxicam & 5 & 12 & 0 & 52 & 69 \\
\hline Other nsNSAID & 14 & 40 & 19 & 0 & 73 \\
\hline
\end{tabular}

\footnotetext{
$\dagger$ Or to September 30, 2005, for patients finishing rofecoxib prescriptions after October 1, 2004. nsNSAID: nonselective nonsteroidal antiinflammatory drug; COX-2: cyclooxygenase inhibitor.
} 
ed hospitalizations in people switching from rofecoxib to nsNSAID, but did find an increase in outpatient visits for peptic ulcer disease ${ }^{11}$.

The following limitations of our study should be considered when interpreting the results. First, although hospitalizations for GI events and endoscopy have been validated as outcome measures ${ }^{16}$, physician visits for GI symptoms may not always be recorded as such, potentially resulting in underestimation of outpatient GI events. Second, the database used to determine drug exposure does not include data regarding nonprescription use of other available NSAID (e.g., ibuprofen) and thus this potential exposure is not accounted for. Third, there is inherent difficulty in characterizing the duration of use of warfarin and corticosteroids due to the nature of the treatment course, which often involves frequent dosing changes, as well as tapering schedules that are not always recorded in the PharmaNet database. This may have resulted in misclassification of a proportion of patients in the regression analysis. Fourth, in the event that patients had overlapping nsNSAID and COX-2 inhibitor prescriptions, we assumed that the first prescription was discontinued, which may underestimate exposures in some patients. Also, although there is an accepted classification scheme for NSAID as either COX-2-selective or nonselective, these drug entities in fact make up a spectrum of enzyme selectivity, and we were unable to account for this variability. Finally, there is some possibility that cotherapy with GI drugs (PPI, $\mathrm{H}_{2}$ antagonists, or misoprostol) or lower doses or intermittent use of NSAID could lead to a reduction in events, thus accounting for a lack of difference between the groups. From Table 1, it appears that patients prescribed nsNSAID have a higher use of concomitant GI drugs than those taking COX-2 inhibitors.

The latter information is supported by the recently published CONDOR trial results, which demonstrated in a straightforward randomized controlled trial of celecoxib versus NSAID that most of the noted adverse events were of bleeding and not ulcers or other complications of ulcers. This information might be helpful for the discussion.

Our report has several strengths, including the methodology used for determining GI events, which has been shown to have high validity ${ }^{16,17}$. In our statistical analysis, a time-dependent Cox model was used, allowing the exposure status to vary with time and thus more accurately represent an individual's changing exposure status ${ }^{18}$. Finally, we conducted sensitivity analyses to measure potential effects of our chosen $125 \%$ exposure time estimate. These analyses showed no effect on HR, indicating that our model was robust with regard to the estimated duration of drug exposure. In terms of generalizability, the databases used for determining drug exposures record all individuals enrolled in the publicly funded BC health insurance program, effectively identifying all patients with RA in the province regardless of source of funding for medications.
We conclude that in this cohort of patients with RA the withdrawal of rofecoxib from the market resulted in a prescribing shift mainly to other COX-2 inhibitors, and did not result in a significant increase in GI events over the following month or year.

\section{REFERENCES}

1. Burnier M. The safety of rofecoxib. Expert Opin Drug Saf 2005;4:491-9.

2. Williams D, Singh M, Hind C. The effect of the withdrawal of rofecoxib on prescribing patterns of COX-2 inhibitors in Scotland. Br J Clin Pharmacol 2006;62:366-8.

3. Barozzi N, Tett SE. What happened to the prescribing of other COX-2 inhibitors, paracetamol and non-steroidal anti-inflammatory drugs when rofecoxib was withdrawn in Australia? Pharmacoepidemiol Drug Saf 2007;16:1184-91

4. Setakis E, Leufkens HG, van Staa TP. Changes in the characteristics of patients prescribed selective cyclooxygenase 2 inhibitors after the 2004 withdrawal of rofecoxib. Arthritis Rheum 2008;59:1105-11

5. Teeling M, O'Connor H, Feely J, Bennett K. What therapies have replaced rofecoxib in Ireland? Br J Clin Pharmacol 2007; 64:536-41.

6. Sukel MP, van der Linden MW, Chen C, Erkens JA, Herings RM. Large-scale stopping and switching treatment with COX-2 inhibitors after the rofecoxib withdrawal. Pharmacoepidemiol Drug Saf 2008;17:9-19.

7. Rahme E, Roussy JP, Lafrance JP, Nedjar H, Morin S. Use of nonsteroidal antiinflammatory drugs: Is there a change in patient risk profile after withdrawal of rofecoxib? J Rheumatol 2011;38:195-202.

8. Taha AS, Angerson WJ, Prasad R, McCloskey C, Blatchford O. Upper gastrointestinal bleeding and the changing use of COX-2 non-steroidal anti-inflammatory drugs and low-dose aspirin. Aliment Pharmacol Ther 2007;26:1171-8.

9. Metcalfe C, Wheeler BW, Gunnell D, Martin RM. International regulatory activity restricting $\mathrm{COX}-2$ inhibitor use and deaths due to gastrointestinal haemorrhage and myocardial infarction. Pharmacoepidemiol Drug Saf 2010;19:778-85.

10. Wheeler BW, Metcalfe C, Gunnell D, Stephens P, Martin RM. Population impact of regulatory activity restricting prescribing of COX-2 inhibitors: ecological study. Br J Clin Pharmacol 2009;68:752-64.

11. Schneeweiss S, Glynn RJ, Avorn J, Mamdani M, Mogun H, Solomon DH. NSAID switching and short-term gastrointestinal outcome rates after the withdrawal of rofecoxib. Pharmacoepidemiol Drug Saf 2009;18:1134-42.

12. Deyo RA, Cherkin DC, Ciol MA. Adapting a clinical comorbidity index for use with ICD-9-CM administrative databases. J Clin Epidemiol 1992;45:613-9.

13. Charlson M, Szatrowski TP, Peterson J, Gold J. Validation of a combined comorbidity index. J Clin Epidemiol 1994;47:1245-51.

14. Quan H, Li B, Saunders LD, Parsons GA, Nilsson CI, Alibhai A, et al. Assessing validity of ICD-9-CM and ICD-10 administrative data in recording clinical conditions in a unique dually coded database. Health Serv Res 2008;43:1424-41.

15. Charlson ME, Pompei P, Ales KL, MacKenzie CR. A new method of classifying prognostic comorbidity in longitudinal studies: Development and validation. J Chronic Dis 1987;40:373-83.

16. Raiford DS, Perez Gutthann S, Garcia Rodriguez LA. Positive predictive value of ICD-9 codes in the identification of cases of complicated peptic ulcer disease in the Saskatchewan hospital automated database. Epidemiology 1996;7:101-4.

17. Cooper GS, Chak A, Lloyd LE, Yurchick PJ, Harper DL, Rosenthal GE. The accuracy of diagnosis and procedural codes for patients with upper GI hemorrhage. Gastrointest Endosc 2000;51:423-6.

18. Barnett A, Graves N. Competing risks models and time-dependent covariates. Crit Care 2008;12:134. 